\title{
sciendo
}

Int. J. of Applied Mechanics and Engineering, 2020, vol.25, No.2, pp.22-39

DOI: $10.2478 /$ ijame-2020-0018

\section{EFFECT OF MAGNETIC FIELD DEPENDENT VISCOSITY ON THE UNSTEADY FERROFLUID FLOW DUE TO A ROTATING DISK}

\author{
A. BHANDARI \\ Department of Mathematics, College of Engineering Studies \\ University of Petroleum and Energy Studies \\ Dehradun, INDIA \\ E-mail: Pankaj.anupam6@gmail.com
}

\begin{abstract}
The effect of magnetic field dependent viscosity on ferrofluid flow due to a rotating disk is studied in the presence of a stationary magnetic field. The results for velocity profiles for various values of MFD viscosity parameter are shown graphically. These results are compared with the ordinary case when the applied magnetic field is absent. Besides, the shear stress on the wall of the disk and its surface is calculated numerically.
\end{abstract}

Key words: ferrofluid, MFD viscosity, rotating risk.

\section{Introduction}

Ferrofluids are stable suspensions of colloidal ferromagnetic particles of the order of $10 \mathrm{~nm}$ in suitable non-magnetic carrier liquids. These colloidal particles are coated with surfactants to avoid their agglomeration. Because of the industrial applications of ferrofluids, the investigation on them fascinated the researchers and engineers vigorously since the last five decades. One of the many fascinating features of the ferrofluids is the prospect of influencing flow by a magnetic field and vice-versa $[1,2]$. Ferrofluids are widely used in the sealing of hard disc drives, rotating x-ray tubes under engineering applications. Sealing of the rotating shafts is the most known application of the magnetic fluids. The major application of ferrofluids in the electrical field is that controlling heat in loudspeakers which makes its life longer and increases the acoustical power without any change in the geometrical shape of the speaker system. Magnetic fluids are used in the contrast medium in X-ray examinations and positioning tamponade for retinal detachment repair in eye surgery. Therefore, ferrofluids play an important role in biomedical applications also. In the presence of the uniform magnetic field, the magnetization characteristics depend on the particle spin but do not depend on the fluid velocity. Convection of ferromagnetic fluid is gaining much importance due to their astounding physical properties.

There are rotationally symmetric flows of the incompressible ferrofluids in the field of fluid mechanics, having all three velocity components; radial, tangential and vertical in space different from zero. In such types of flow, the variables are independent of the angular coordinates. Detail accounts of magneto viscous effects in ferrofluids have been given in a monograph by Odenbach [3]. Flow for an incompressible ferrofluid, the plate is subjected to the magnetic field $\left[H_{r}, 0, H_{z}\right]$ using, Neuringer-Rosensweig model [4]. This model has been used by Verma et al. $[5,6,7]$ for solving paramagnetic Couette flow, helical flow with heat conduction and flow through a porous annulus. Rosensweig [8] has given an authoritative introduction to the research on magnetic liquids in his monograph and studied the effect of magnetization, resulting in interesting information.

A study of flow within the boundary layer and its effect on the general flow around the body, in detail, are given in Schlichting [9]. Karman's [10] rotating disc problem is extended to the case of flow started impulsively from rest and also the steady-state is solved to a higher degree of accuracy than 
previously done by a simple analytical method which neglects the resembling difficulties in Cochran's [11] well-known solution. The pioneering study of ordinary viscous fluid flow due to the infinite rotating disc was carried by Von Karman. He introduced the famous transformation which reduces the governing partial differential equations into ordinary differential equations. Cochran obtained asymptotic solutions for the steady hydrodynamic problem formulated by Von Karman. Benton [12] improved Cochran's solutions and solved the unsteady case. Attia [13] studied the unsteady state in the presence of an applied uniform magnetic field. The steady flow of ordinary viscous fluid due to the rotating disc with uniform high suction was studied by Mithal [14]. Attia [15] discussed flow due to an infinite disk rotating in the presence of an axial uniform magnetic field by taking Hall effect into consideration.

Using linear instability analysis, Venkatasubramanian and Kaloni [16] discussed the effects of rotation on the onset of convection in a horizontal layer of ferrofluids rotating about its vertical axis, heated from below and in the presence of a uniform vertical magnetic field. The effect of an alternating uniform magnetic field on convection in a horizontal layer of a ferrofluid within the framework of a quasi-stationary approach is studied by Belyaev [17].

The effect of the magnetic field along the vertical axis on thermo-convective instability in a ferromagnetic fluid saturating a rotating porous medium has been studied by Sekar et al. [18] by using the Darcy model. Attia [19] studied the steady flow of an incompressible viscous fluid above an infinite rotating disk in a porous medium with heat transfer and also discussed the effect of porosity of medium on the velocity and temperature distribution. Frusteri and Osalusi [20] examined the laminar convective and slip flow of an electrically conducting Newtonian fluid with variable properties over a rotating porous disk.

In general, magnetization is a function of the magnetic field, temperature and density of the fluid. This leads to convection of ferrofluid in the presence of the magnetic field gradient. Nanjundappa et al. [21] studied Benard-Marangoni Ferroconvection in a ferrofluid layer in the presence of a uniform vertical magnetic field with magnetic field dependent (MFD) viscosity. Ram et al. [22] solved the non-linear differential equations under Neuringer-Rosensweig model for ferrofluid flow by using power series approximations and discussed the effect of magnetic field-dependent viscosity on the velocity components and pressure profile. Negative viscosity effects due to an alternating magnetic field are studied by Ram and Bhandari [23].

In the present study, the effect of magnetic field dependent viscosity on unsteady ferrofluid flow due to a rotating disk is studied in the presence of a stationary magnetic field. When we apply the externally applied field on the ferrofluid, the viscosity of the fluid increases and depend on the intensity of the magnetic field. We take cylindrical coordinates $r, \theta, z$, where the $z$-axis is normal to the plane and this axis is considered as the axis of rotation. Let $v_{r}, v_{\theta}$ and $v_{z}$ are the radial, tangential and axial components of velocity, respectively and the disk is rotating with uniform angular velocity $\omega$. Neruinger-Rosensweig model is used in the problem formulation and this problem is considered with the assumptions that the fluid and the disk are both electrically non-conducting, Magnetic field affects viscosity only and flow is taken axisymmetric.

\section{Formulation of the problem}

The constitutive set of equations is as follows:

The equation of momentum

$$
\rho\left[\frac{\partial v}{\partial t}+(v \cdot \nabla) v\right]=-\nabla p+\mu_{0}(M . \nabla) H+\mu(1+\delta . B) \nabla^{2} v
$$

\section{The equation of continuity}

$$
\nabla \cdot v=0 \text {. }
$$




\section{Maxwell equations}

$$
\nabla \times H=0, \quad \nabla .(H+M)=0 \quad \text { with } \quad M=\chi H .
$$

Here $\rho$ is the density of the fluid, $v$ is the fluid velocity, $p$ is the pressure, $\boldsymbol{M}$ is the magnetization, $\boldsymbol{H}$ is the magnetic field intensity, $\mu_{0}$ is the permeability of free space, $\mu$ is the reference viscosity of the fluid, $\delta$ is the linear measurement in the viscosity due to applied magnetic field, $\boldsymbol{B}$ is the magnetic induction, $\chi$ is the magnetic susceptibility, $t$ is the time.

For the flow due to a rotating disk, the Eqs (2.1) and (2.2) can be written in the cylindrical form as

$$
\begin{aligned}
& -\frac{1}{\rho} \frac{\partial \tilde{p}}{\partial r}+v_{I}\left[\frac{\partial^{2} v_{r}}{\partial r^{2}}+\frac{\partial}{\partial r}\left(\frac{v_{r}}{r}\right)+\frac{\partial^{2} v_{r}}{\partial z^{2}}\right]=\left[\frac{\partial v_{r}}{\partial t}+v_{r} \frac{\partial v_{r}}{\partial r}+v_{z} \frac{\partial v_{r}}{\partial z}-\frac{v_{\theta}^{2}}{r}\right] \\
& v_{I}\left[\frac{\partial^{2} v_{\theta}}{\partial r^{2}}+\frac{\partial}{\partial r}\left(\frac{v_{\theta}}{r}\right)+\frac{\partial^{2} v_{\theta}}{\partial z^{2}}\right]=\left[\frac{\partial v_{\theta}}{\partial t}+v_{r} \frac{\partial v_{\theta}}{\partial r}+v_{z} \frac{\partial v_{\theta}}{\partial z}+\frac{v_{r} v_{\theta}}{r}\right] \\
& -\frac{1}{\rho} \frac{\partial \tilde{p}}{\partial z}+v_{1}\left[\frac{\partial^{2} v_{z}}{\partial r^{2}}+\frac{1}{r} \frac{\partial v_{z}}{\partial r}+\frac{\partial^{2} v_{z}}{\partial z^{2}}\right]=\left[\frac{\partial v_{z}}{\partial t}+v_{r} \frac{\partial v_{z}}{\partial r}+v_{z} \frac{\partial v_{z}}{\partial z}\right] \\
& \frac{\partial v_{r}}{\partial r}+\frac{v_{r}}{r}+\frac{\partial v_{z}}{\partial z}=0 .
\end{aligned}
$$

In Eqs (2.4) and (2.6), $\tilde{p}$ is the reduced pressure due to the magnetization force i.e. $-\nabla \tilde{p}=-\nabla p+\mu_{0}(M . \nabla) H$, and $v_{1}=\frac{\mu(1+\delta . B)}{\rho}$ is the magnetic field dependent viscosity.

If the magnetization and the magnetic field intensity are parallel to each other, then the reduced pressure can be calculated as

$$
\tilde{p}=p-\int_{0}^{H} M d H^{\prime}=p-\int_{0}^{H} \chi H^{\prime} d H^{\prime}=p-\chi\left[\frac{H^{\prime 2}}{2}\right]_{0}^{H}=p-\chi \frac{H^{2}}{2}
$$

For the flow due to a rotating disk about $z$-axis with a constant angular velocity $\omega$, the boundary conditions used by both Attia and Ariel $[15,24]$ are given as follows

at

at

$$
\begin{aligned}
& z=0 ; \quad v_{r}=0, \quad v_{\theta}=r \omega, \quad v_{z}=0 \quad \text { at } \quad z \rightarrow \infty ; \quad v_{r}=0, \quad v_{\theta}=0 ; \\
& t=0, \quad v_{r}=0, \quad v_{\theta}=0, \quad v_{z}=0 .
\end{aligned}
$$

Now, we use the similarity transformation to convert Eqs (2.4)-(2.7) in dimensionless form as [10, 11]

$$
v_{r}=r \omega E(\beta), \quad v_{\theta}=r \omega F(\beta), \quad v_{z}=\sqrt{v \omega} G(\beta), \quad \tilde{p}=\rho \omega v P(\beta), \quad \beta=z \sqrt{\frac{\omega}{v}}, \quad t_{d}=\omega t
$$


Here $\beta$ is the dimensionless axial distance from the disk, $E(\beta), F(\beta), G(\beta)$ are the dimensionless components of radial, tangential, axial velocities respectively, $P(\beta)$ is the dimensionless pressure profile, $v$ is the kinematic viscosity and $t_{d}$ is the dimensionless time parameter.

Using similarity transformations Eq.(2.10), Eqs (2.4)-(2.7) are transformed to the following differential equations

$$
\begin{aligned}
& \frac{\partial E}{\partial t_{d}}-k \frac{\partial^{2} E}{\partial \beta^{2}}+G \frac{\partial E}{\partial \beta}+E^{2}-F^{2}=0, \\
& \frac{\partial F}{\partial t_{d}}-k \frac{\partial^{2} F}{\partial \beta^{2}}+G \frac{\partial F}{\partial \beta}+2 E F=0 \\
& \frac{\partial G}{\partial t_{d}}+\frac{\partial P}{\partial \beta}-k \frac{\partial^{2} G}{\partial \beta^{2}}+G \frac{\partial G}{\partial \beta}=0 \\
& \frac{\partial G}{\partial \beta}+2 E=0 .
\end{aligned}
$$

Here $k$ denotes the magnetic field dependent (MFD) viscosity parameter and mathematically it can be written as, $\frac{v_{1}}{v}=1+\delta . B=k$. In the absence of magnetic field, the magnetic fluid behaves as an ordinary fluid which follows Newton's law, however, in the presence of an externally applied magnetic field, viscous properties are controlled by the strength of the applied magnetic field.

Here, the transformed boundary conditions are

$$
\begin{aligned}
& E\left(t_{d}, 0\right)=0, \quad F\left(t_{d}, 0\right)=1, \quad G\left(t_{d}, 0\right)=0 ; \quad E\left(t_{d}, \infty\right)=0, \quad F\left(t_{d}, \infty\right)=0 \text { and } \\
& E(0, \beta)=0, \quad F(0, \beta)=0, \quad G(0, \beta)=0 .
\end{aligned}
$$

\section{Solution procedure}

Equations (2.11)-(2.14) are nonlinear coupled partial differential equations. These equations are solved with the help of Flex PDE after a suitable coordinate transformation. Here, if we solve (2.11)-(2.14) directly, the solution oscillates due to discontinuity which occurs between initial and boundary conditions in Eq.(2.15). Therefore, the Eqs (2.11)-(2.14) are transformed into appropriate form by taking a suitable coordinate transformation $\delta=\beta / 2 \sqrt{t_{d}}$ as suggested by Attia in the similar kind of problems [13]. Now, the transformed equations are

$$
\begin{aligned}
& \frac{\partial E}{\partial t_{d}}-\frac{\delta}{2 t_{d}} \frac{\partial E}{\partial \delta}-\frac{k}{4 t_{d}} \frac{\partial^{2} E}{\partial \delta^{2}}+G \frac{1}{2 \sqrt{t_{d}}} \frac{\partial E}{\partial \delta}+E^{2}-F^{2}=0, \\
& \frac{\partial F}{\partial t_{d}}-\frac{\delta}{2 t_{d}} \frac{\partial F}{\partial \delta}-\frac{k}{4 t_{d}} \frac{\partial^{2} F}{\partial \delta^{2}}+G \frac{1}{2 \sqrt{t_{d}}} \frac{\partial F}{\partial \delta}+2 E F=0,
\end{aligned}
$$




$$
\frac{\partial G}{\partial \delta}+4 \sqrt{t_{d}} E=0
$$

The above equations are solved in Flex PDE for various values of $k$ and $t_{d}$ these results are shown graphically here. If the flow is considered steady, the Eqs (2.11)-(2.14) reduces to ordinary nonlinear coupled differential equations as

$$
\begin{aligned}
& k \frac{d^{2} E}{d \beta^{2}}-G(\beta) \frac{d E}{d \beta}-E^{2}(\beta)+F^{2}(\beta)=0, \\
& k \frac{d^{2} F}{d \beta^{2}}-G(\beta) \frac{d F}{d \beta}-2 E(\beta) F(\beta)=0, \\
& \frac{d P}{d \beta}-k \frac{d^{2} G}{d \beta^{2}}+G(\beta) \frac{d G}{d \beta}=0, \\
& G^{\prime}(\beta)+2 E(\beta)=0 .
\end{aligned}
$$

Here, $\quad E(0)=0, \quad F(0)=1, G(0)=0 \quad$ and $\quad E(\infty)=F(\infty)=0$.

The resulting system of equations is solved numerically by applying a shooting iteration technique combined with a fourth-order Runge-Kutta method. Therefore, we reduce the Eqs (3.4)-(3.7) into first-order differential equations by using the following transformation as

$$
E(\beta) \rightarrow y_{1}(\beta), \quad \frac{d E}{d \beta} \rightarrow y_{2}(\beta), \quad F(\beta) \rightarrow y_{3}(\beta), \quad \frac{d F}{d \beta} \rightarrow y_{4}(\beta), \quad G(\beta) \rightarrow y_{5}(\beta) .
$$

Let $y_{2}(0)=a$ and $y_{4}(0)=b$, we get an Initial Value Problem 1. We will find $a$ and $b$ later.

\section{i. Initial Value Problem 1}

$$
\begin{aligned}
& \frac{d y_{1}}{d \beta}=y_{2} ; \quad y_{1}(0)=0, \\
& \frac{d y_{2}}{d \beta}=\frac{1}{k}\left\{y_{2} y_{5}+y_{1}^{2}-y_{3}^{2}\right\} ; \quad y_{2}(0)=a, \\
& \frac{d y_{3}}{d \beta}=y_{4} ; \quad y_{3}(0)=1, \\
& \frac{d y_{4}}{d \beta}=\frac{1}{k}\left\{2 y_{1} y_{3}+y_{5} y_{4}\right\} ; \quad y_{4}(0)=b,
\end{aligned}
$$




$$
\frac{d y_{5}}{d \beta}=-2 y_{1} ; \quad y_{5}(0)=0
$$

Differentiating Eqs (3.10)-(3.14) partially with respect to $a$ as

$$
Y_{i}=\frac{\partial y_{i}}{\partial a}, \quad \text { for } \quad i=1,2,3,4,5
$$

We get the Initial Value Problem 2 as:

\section{ii. Initial Value Problem 2}

$$
\begin{aligned}
& \frac{d Y_{1}}{d \beta}=Y_{2} ; \quad Y_{1}(0)=0, \\
& \frac{d Y_{2}}{d \beta}=\frac{1}{k}\left\{Y_{5} y_{2}+y_{5} Y_{2}+2 y_{1} Y_{1}-2 y_{3} Y_{3}\right\} ; \quad Y_{2}(0)=1, \\
& \frac{d Y_{3}}{d \beta}=Y_{4} ; \quad Y_{3}(0)=0, \\
& \frac{d Y_{4}}{d \beta}=\frac{1}{k}\left\{2 Y_{1} y_{3}+2 y_{1} Y_{3}+Y_{5} y_{4}+y_{5} Y_{4}\right\} ; \quad Y_{4}(0)=0, \\
& \frac{d Y_{5}}{d \beta}=-2 Y_{1} ; \quad Y_{5}(0)=0 .
\end{aligned}
$$

Again, differentiating Eqs (3.10)-(3.14) partially with respect to $b$ such as

$$
Z_{i}=\frac{\partial y_{i}}{\partial b}, \quad \text { for } \quad i=1,2,3,4,5 \text {. }
$$

We get the Initial Value Problem 3 as:

\section{iii. Initial Value Problem 3}

$$
\begin{aligned}
& \frac{d Z_{1}}{d \beta}=Z_{2} ; \quad Z_{1}(0)=0, \\
& \frac{d Z_{2}}{d \beta}=\frac{1}{k}\left\{Z_{5} y_{2}+y_{5} Z_{2}+2 y_{1} Z_{1}-2 y_{3} Z_{3}\right\} ; \quad Z_{2}(0)=0, \\
& \frac{d Z_{3}}{d \beta}=Z_{4} ; \quad Z_{3}(0)=0,
\end{aligned}
$$




$$
\begin{aligned}
& \frac{d Z_{4}}{d \beta}=\frac{1}{k}\left\{2 Z_{1} y_{3}+2 y_{1} Z_{3}+Z_{5} y_{4}+y_{5} Z_{4}\right\} ; \quad Z_{4}(0)=1, \\
& \frac{d Z_{5}}{d \beta}=-2 Z_{1} ; \quad Z_{5}(0)=0 .
\end{aligned}
$$

Let $\quad f_{l}\left(\infty ; a_{n}, b_{n}\right)=y_{1}\left(\infty ; a_{n}, b_{n}\right)-y_{1}(\infty)=y_{1}\left(\infty ; a_{n}, b_{n}\right)-0=y_{1}\left(\infty ; a_{n}, b_{n}\right)$

and

$$
f_{2}\left(\infty ; a_{n}, b_{n}\right)=y_{3}\left(\infty ; a_{n}, b_{n}\right)-y_{3}(\infty)=y_{3}\left(\infty ; a_{n}, b_{n}\right)-0=y_{3}\left(\infty ; a_{n}, b_{n}\right) .
$$

Now, we can find $a$ and $b$ as follows

$$
\begin{aligned}
& {\left[\begin{array}{l}
a_{n+1} \\
b_{n+1}
\end{array}\right]=\left[\begin{array}{l}
a_{n} \\
b_{n}
\end{array}\right]-\left[\begin{array}{ll}
\frac{\partial f_{1}}{\partial a} & \frac{\partial f_{1}}{\partial b} \\
\frac{\partial f_{2}}{\partial a} & \frac{\partial f_{2}}{\partial b}
\end{array}\right]_{a_{n}, b_{n}}^{-1}\left[\begin{array}{l}
f_{1} \\
f_{2}
\end{array}\right]_{a_{n}, b_{n}},} \\
& {\left[\begin{array}{l}
a_{n+1} \\
b_{n+1}
\end{array}\right]=\left[\begin{array}{l}
a_{n} \\
b_{n}
\end{array}\right]-\left[\begin{array}{ll}
\frac{\partial y_{1}}{\partial a} & \frac{\partial y_{1}}{\partial b} \\
\frac{\partial y_{3}}{\partial a} & \frac{\partial y_{3}}{\partial b}
\end{array}\right]_{a_{n}, b_{n}}^{-1}\left[\begin{array}{l}
f_{1} \\
f_{2}
\end{array}\right]_{a_{n}, b_{n}}}
\end{aligned} .
$$

Here $a$ and $b$ can be chosen positive or negative and depends on the values of $E$ and $F$. In our problem, $E$ increases for increasing values of $\beta$, however, $F$ decreases for increasing values of $\beta$. Therefore, $a$ and $b$ are taken positive and negative, respectively.

From Eq.(3.6), the reduced pressure can be calculated for different values of $k$ as

$$
P(\beta)-P_{0}=k G^{\prime}(\beta)-\frac{1}{2} G^{2}(\beta)
$$

where $P_{0}$ is the constant of integration and represents the initial pressure.

The boundary layer displacement thickness is calculated as

$$
d=\frac{1}{r \omega} \int_{0}^{\infty} v_{\theta} d z=\int_{0}^{\infty} F(\alpha) d \alpha
$$

The expressions for shear stress on the wall of the disk $\left(\tau_{w}\right)$ and its surface $\left(\tau_{s}\right)$ are as follows

$$
\tau_{w}=\mu\left[\frac{\partial v_{\theta}}{\partial z}+\frac{1}{r} \frac{\partial v_{z}}{\partial \theta}\right]_{z=0}, \quad \tau_{s}=\mu\left[\frac{\partial v_{z}}{\partial r}+\frac{\partial v_{r}}{\partial z}\right]_{z=0} .
$$

By using similarity transformation, the skin friction coefficient in the tangential direction $\left(c_{w}\right)$ and in the radial direction $\left(c_{s}\right)$ can be calculated as

$$
\frac{\tau_{w}}{\rho r\left(v \omega^{3}\right)^{\frac{1}{2}}}=c_{w}=F^{\prime}(0), \quad \frac{\tau_{s}}{\rho r\left(v \omega^{3}\right)^{\frac{1}{2}}}=c_{s}=E^{\prime}(0)
$$


Table 1. Skin friction coefficients $c_{w}$ and $c_{s}$ for various values of $k$.

\begin{tabular}{|c|c|c|c|c|}
\hline & $k=1$ & $k=1.1$ & $k=1.2$ & $k=1.3$ \\
\hline$c_{w}$ & 0.6179 & 0.5888 & 0.5634 & 0.5409 \\
\hline$c_{S}$ & 0.5042 & 0.4805 & 0.4596 & $0 . .4411$ \\
\hline
\end{tabular}

\section{Results and discussion}

Figures 1-4 shows the radial velocity profile for various values of the time $t_{d}$ and magnetic field dependent (MFD) viscosity parameter $k$. For $k=1$, the problem reduces to an ordinary case where there is no effect of field-dependent viscosity on the radial velocity profile as shown in Fig.1. In this case, the fluid reaches the steady-state region faster than Fig.2 and much faster than Figs 3, 4. These results indicate that the flow of the fluid is radially outwards throughout the motion since, in all the cases, the radial velocity remains positive. It also clear from the figure that the radial velocity gets higher peak values for $t_{d}=1,3$ in comparison to $t_{d}=0.5,5$. However, the variable viscosity parameter $k$ also increases the peak value of the radial velocity.

Figure 13 is plotted for a steady flow of ferrofluid, i.e. $\frac{\partial}{\partial t}=0$, for various values of $k$. Here at $k=1$ the problem reduces to the ordinary case $[11,12]$. This result indicates that for $k=1$, the radial velocity converges to the steady-state region faster in comparison to ferrofluid.

Figures 5-8 represents the tangential velocity profile. It is clear from the results that, the MFD viscosity parameter does not have much impact on the tangential velocities. However, at $t_{d}=0.5$, the tangential velocity reaches the steady-state region faster than at $t_{d}=1$ and much faster than at $t_{d}=3,5$. In this problem, the disk is rotating with the uniform angular velocity $\omega$ at $z=0$, therefore, the tangential velocity near the disk is maximum since the flow of fluid is influenced by the disk. However, at far from the disk, it decreases continuously, and finally reaches the steady-state region.

In case of the steady flow of ferrofluid, Fig.14 indicate the behaviour of tangential velocity for various values of at $k=1$, the problem reduces to the ordinary viscous flow due to a rotating disk and the results marches with Cochran [11]. However, for $k=1.1,1 \cdot 2,1.3$, the effect of MFD viscosity parameter can be seen clearly.

Figures 9-12 depicts the axial velocity profile. Figure 9 is obtained for ordinary viscous flow where there is no effect of MFD viscosity parameter on the axial velocity. These figures indicate that at $t_{d}=3$, the axial velocity gets large axial velocity at a large distance from the disk in comparison to $t_{d}=0.5,1,5$. For increasing the values of the parameter $k$ increases the axial velocity. Negative values of the axial velocity reflect that the flow is towards the disk. In all the cases, the axial velocity gets a finite value at far from the disk.

If the flow is steady, the axial velocity for various values of $k$ taking the form as shown in Fig.15. In the case of MFD viscosity, ferrofluid gets large axial velocity in comparison to an ordinary viscous fluid, however, a stationary magnetic field is applied on the fluid.

Figure 16 shows the pressure profile for different values of $k$. It is the reduced pressure due to the magnetization force. The original pressure can be determined if the strength of the magnetization force is known. In the case of ferrofluid, the pressure gets higher values in comparison to ordinary viscous fluid with increasing the dimensionless axial distance $\beta$. At large distances from the disk, where the fluid is free from the influence of the disk, the fluid gets constant pressure.

We have also calculated the boundary layer displacement thickness here. In the ordinary case, the thickness of the boundary layer is 1.2297, however, for $k=1.1,1.2,1.3$ the boundary layer thickness is $1.2819,1.3311,1.3931$, respectively. These values indicate that the fluid is thickened due to variable viscosity. The thickness of the boundary layer is calculated by Simpson's One-Third Rule of numerical integration. 
Further, the skin friction on the wall of the disk and its surface is calculated numerically. For increasing values of MFD viscosity parameter, the tangential and the radial skin friction coefficients decrease.

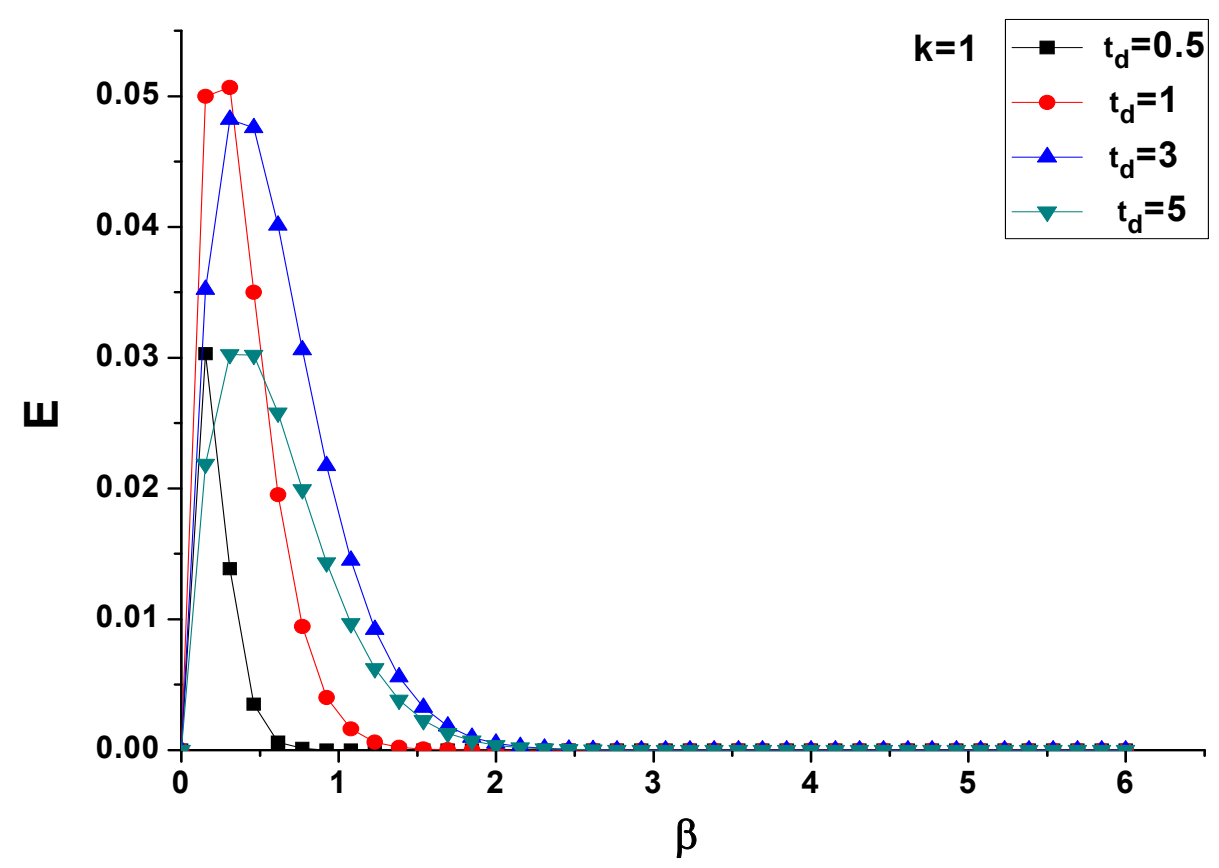

Fig.1. Radial velocity profile for various values of time at $\boldsymbol{k}=1$.

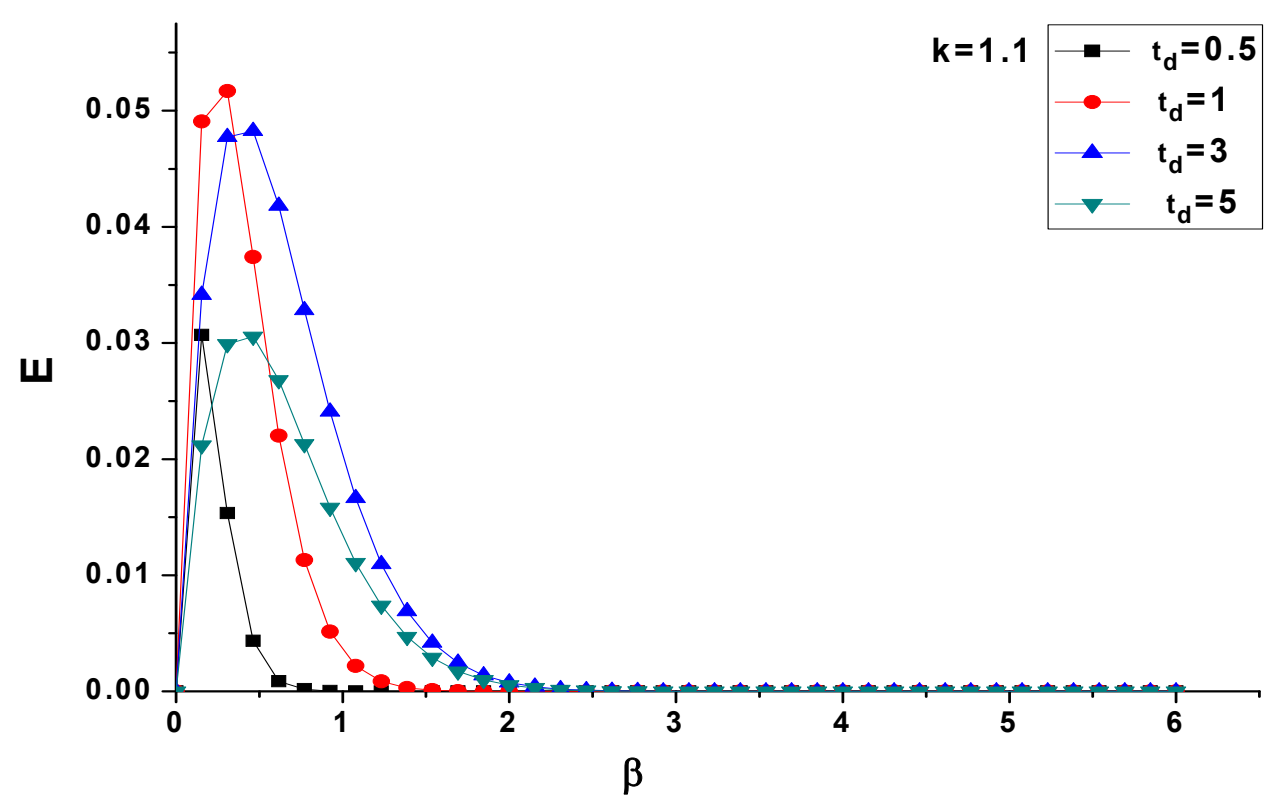

Fig.2. Radial velocity profile for various values of time at $\boldsymbol{k}=1.1$. 


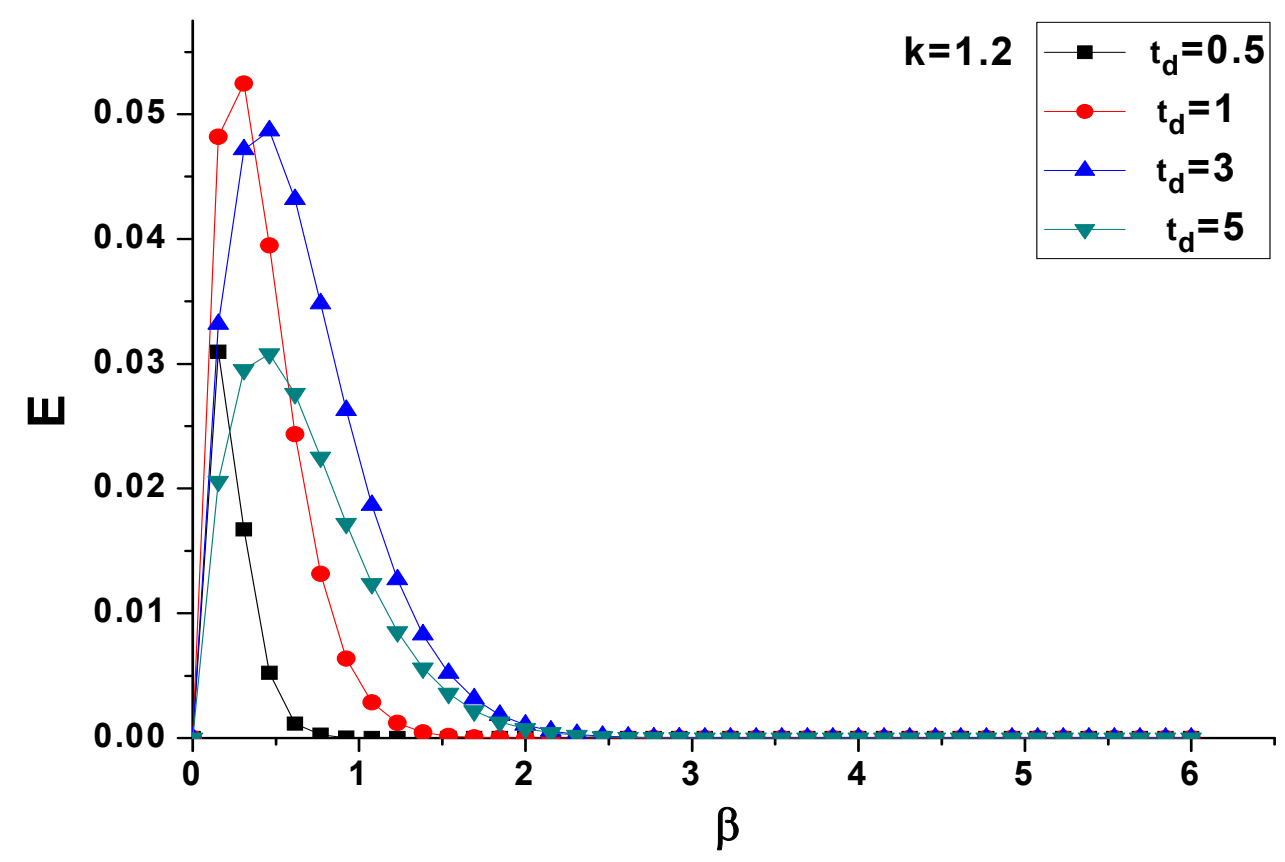

Fig.3. Radial velocity profile for various values of time at $\boldsymbol{k}=1.2$.

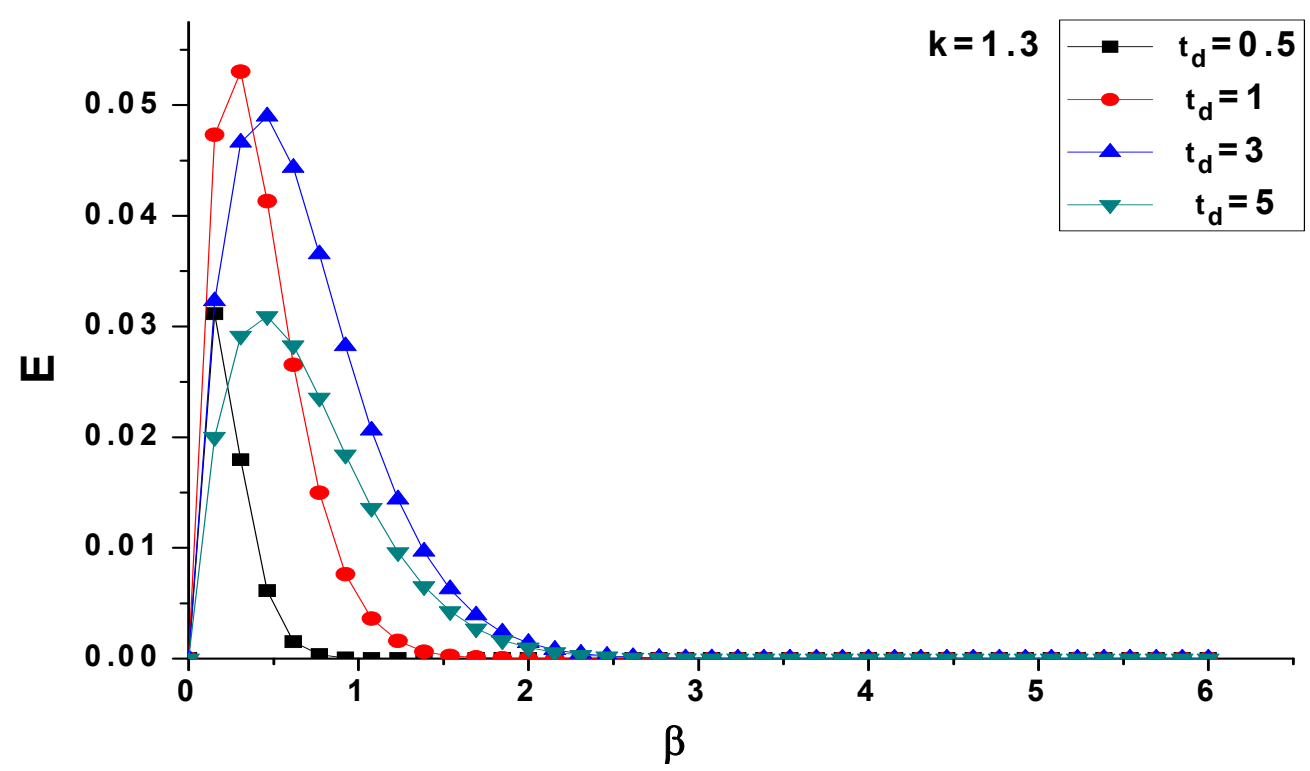

Fig.4. Radial velocity profile for various values of time at $\boldsymbol{k}=1.3$. 


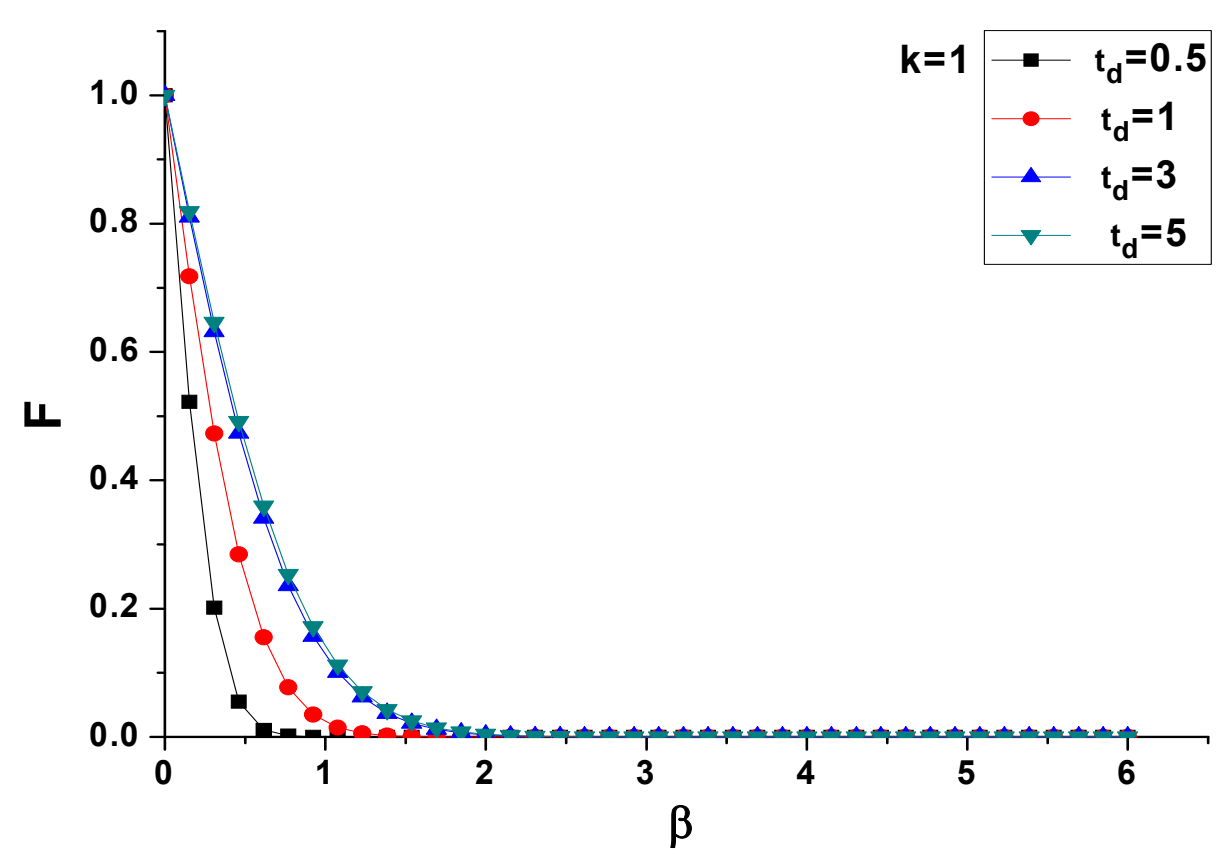

Fig.5. Tangential velocity profile for various values of time at $\boldsymbol{k}=1$.

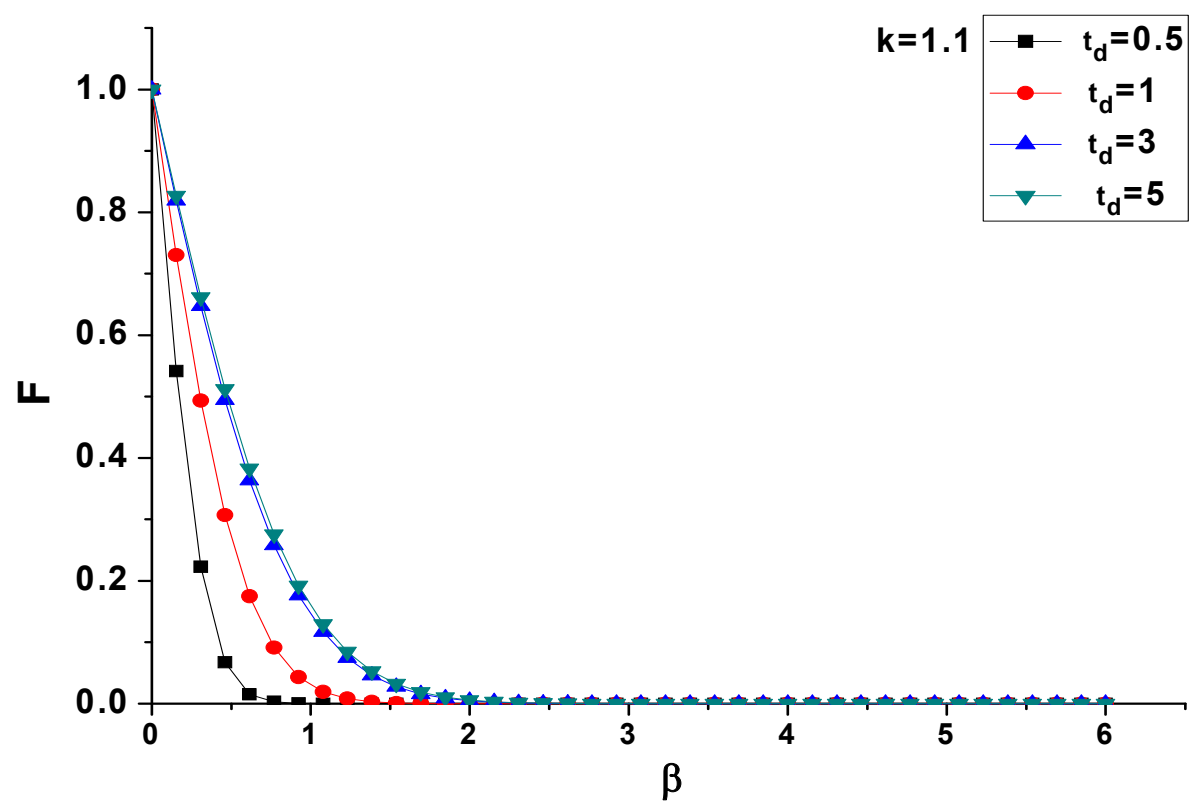

Fig.6. Tangential velocity profile for various values of time at $\boldsymbol{k}=1.1$. 


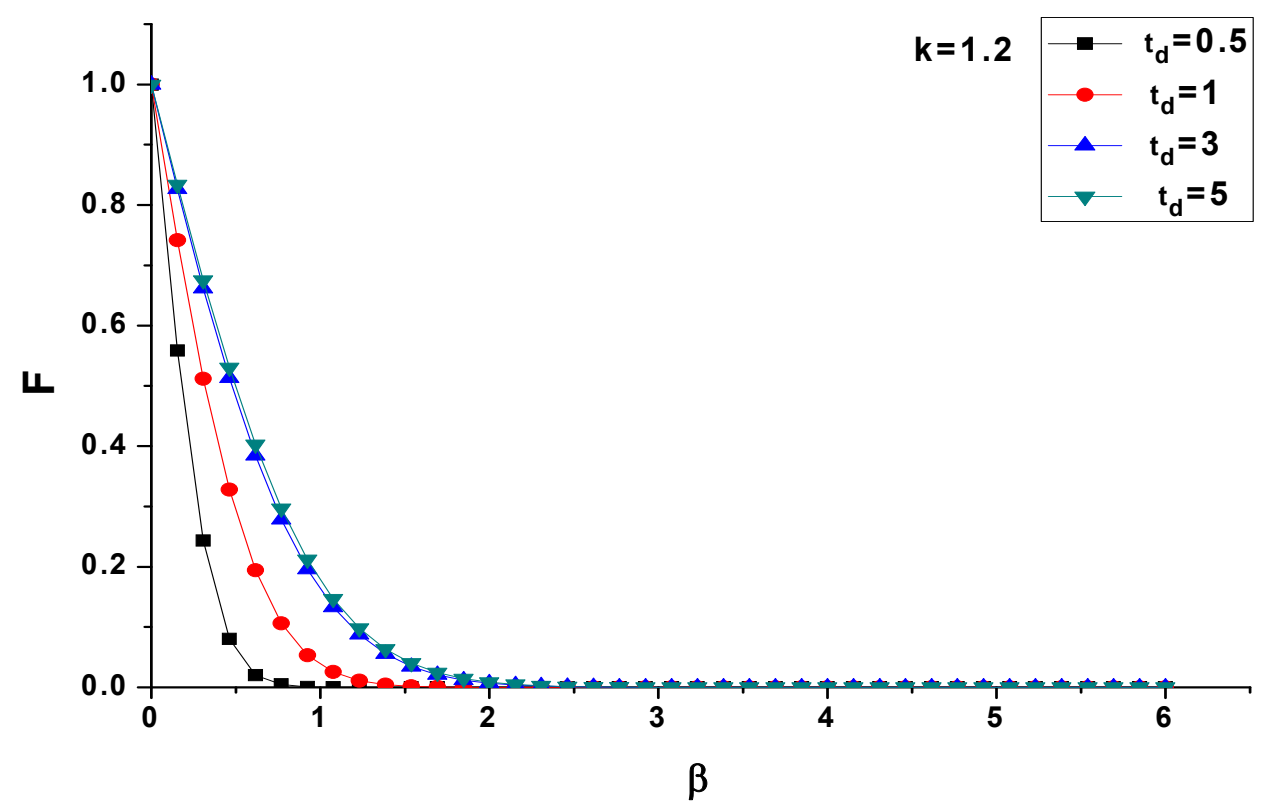

Fig.7. Tangential velocity profile for various values of time at $\boldsymbol{k}=1.2$.

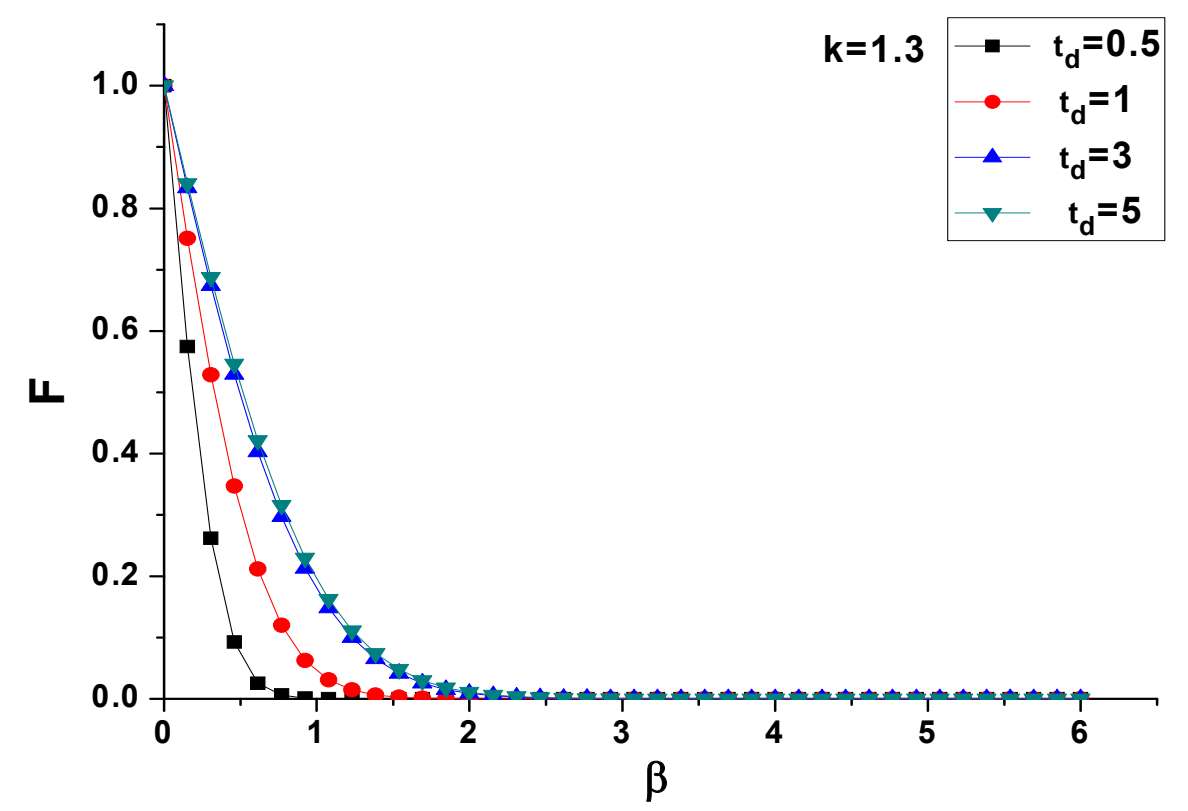

Fig.8. Tangential velocity profile for various values of time at $\boldsymbol{k}=1.3$. 


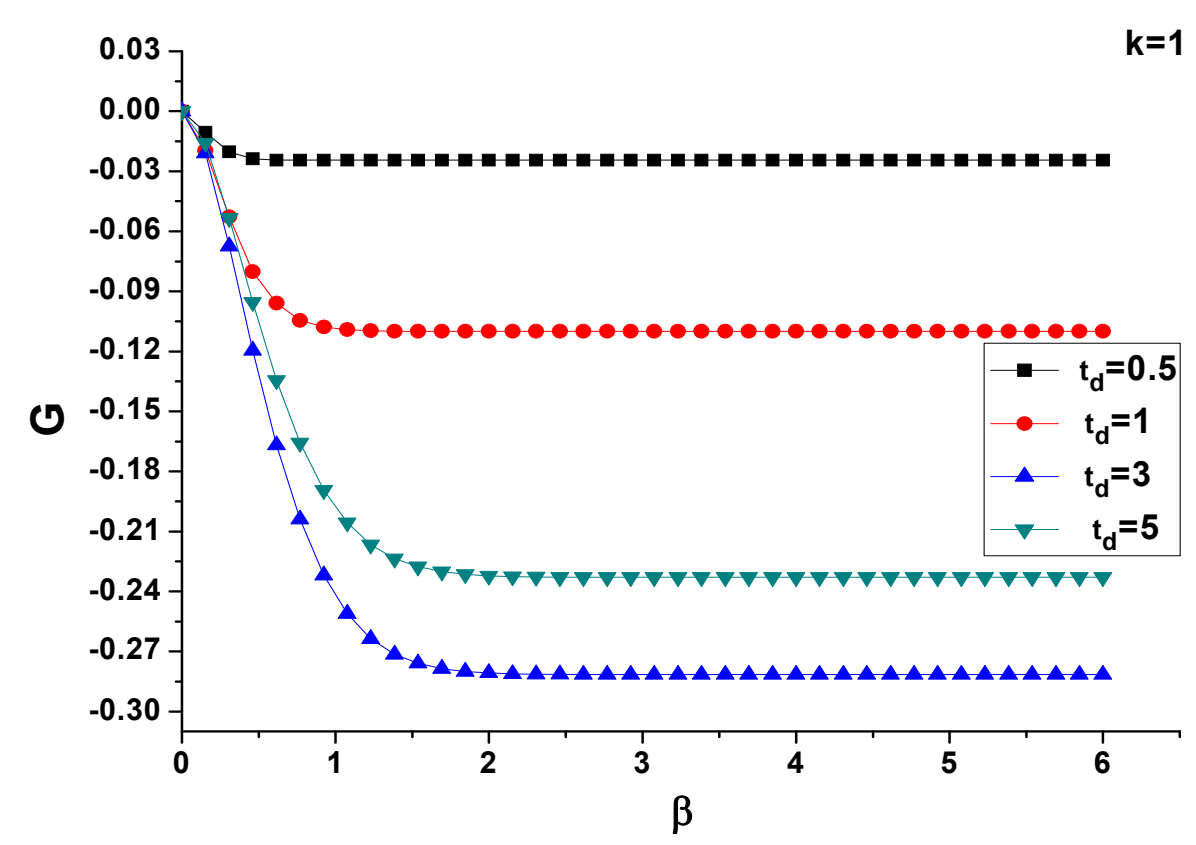

Fig.9. Axial velocity profile for various values of time at $\boldsymbol{k}=1$.

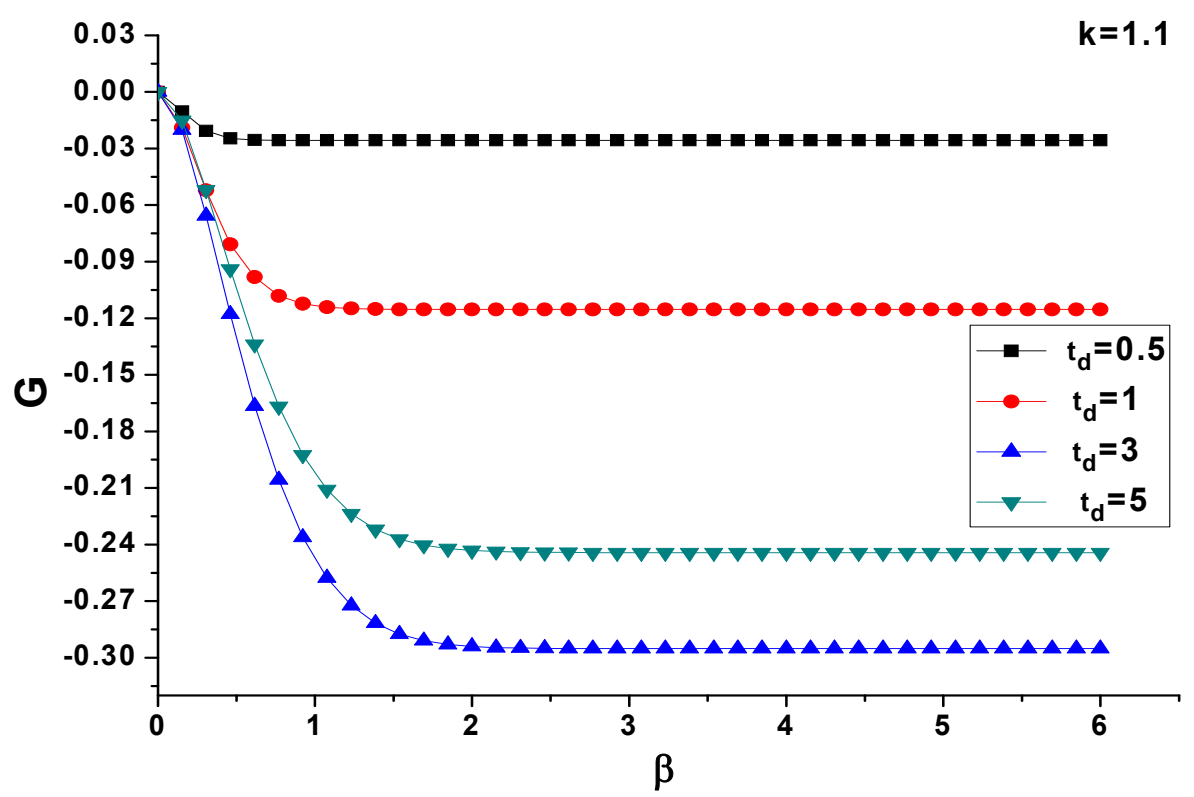

Fig.10. Axial velocity profile for various values of time at $\boldsymbol{k}=1.1$. 


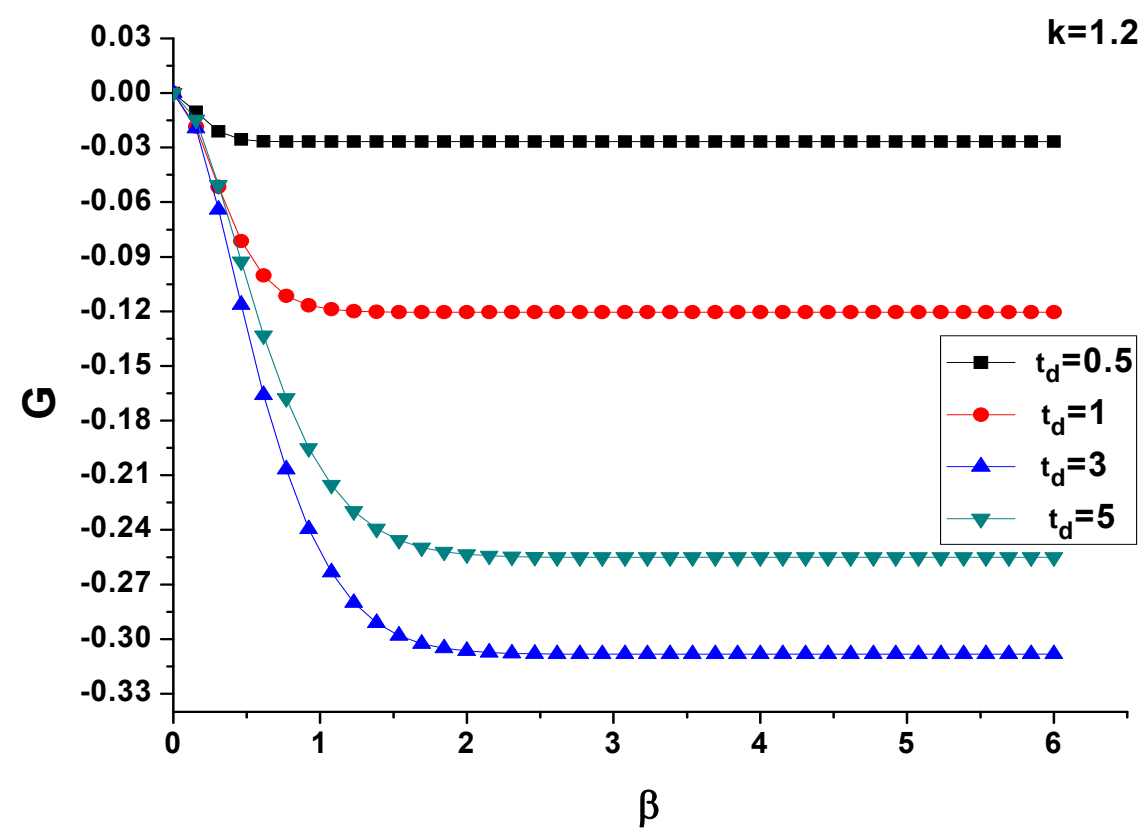

Fig.11. Axial velocity profile for various values of time at $\boldsymbol{k}=1.2$.

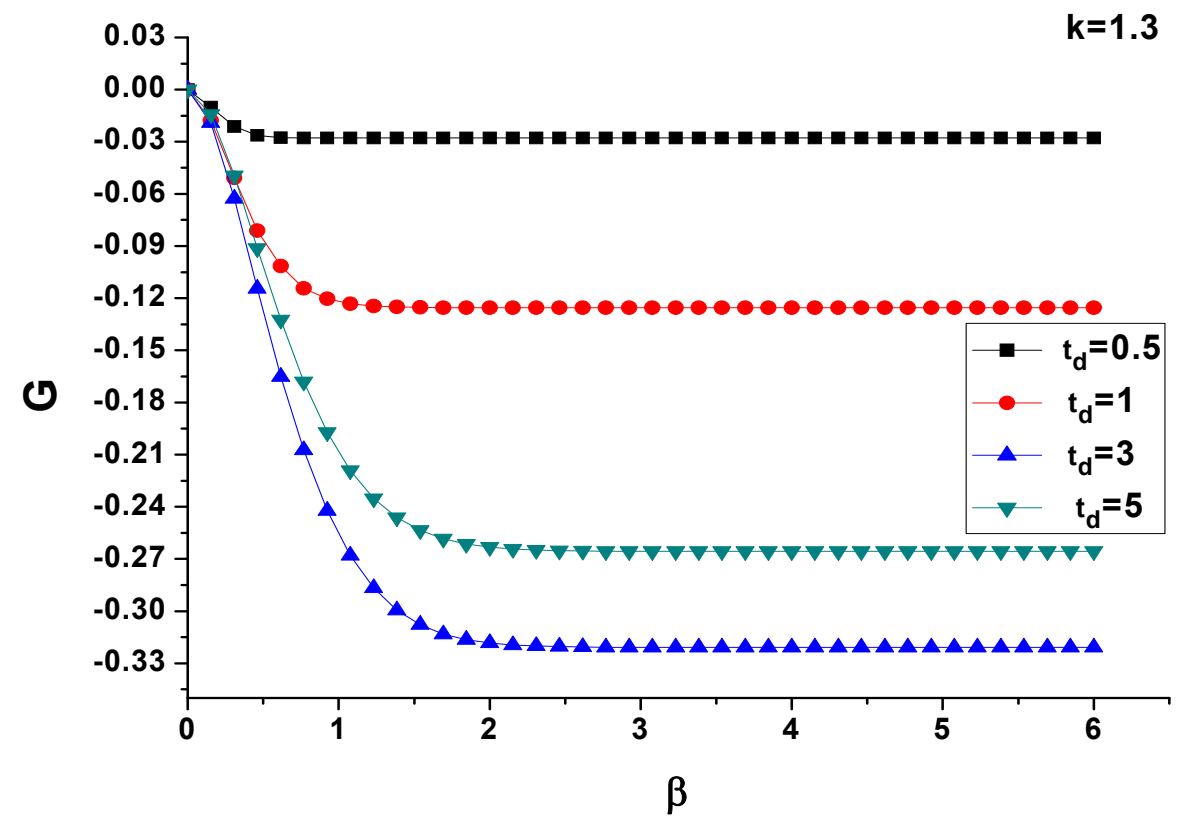

Fig.12. Axial velocity profile for various values of time at $\boldsymbol{k}=1.3$. 


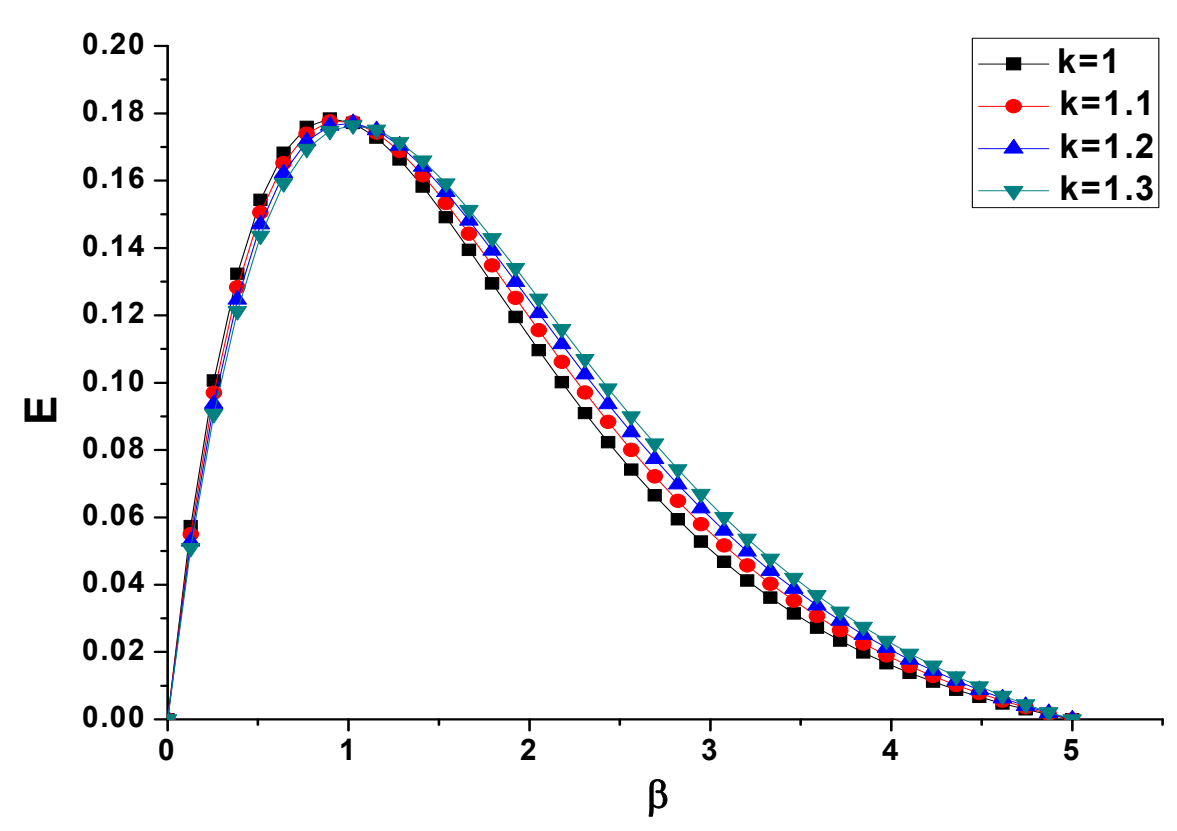

Fig.13. Steady-state radial velocity profile for various values of $\boldsymbol{k}$.

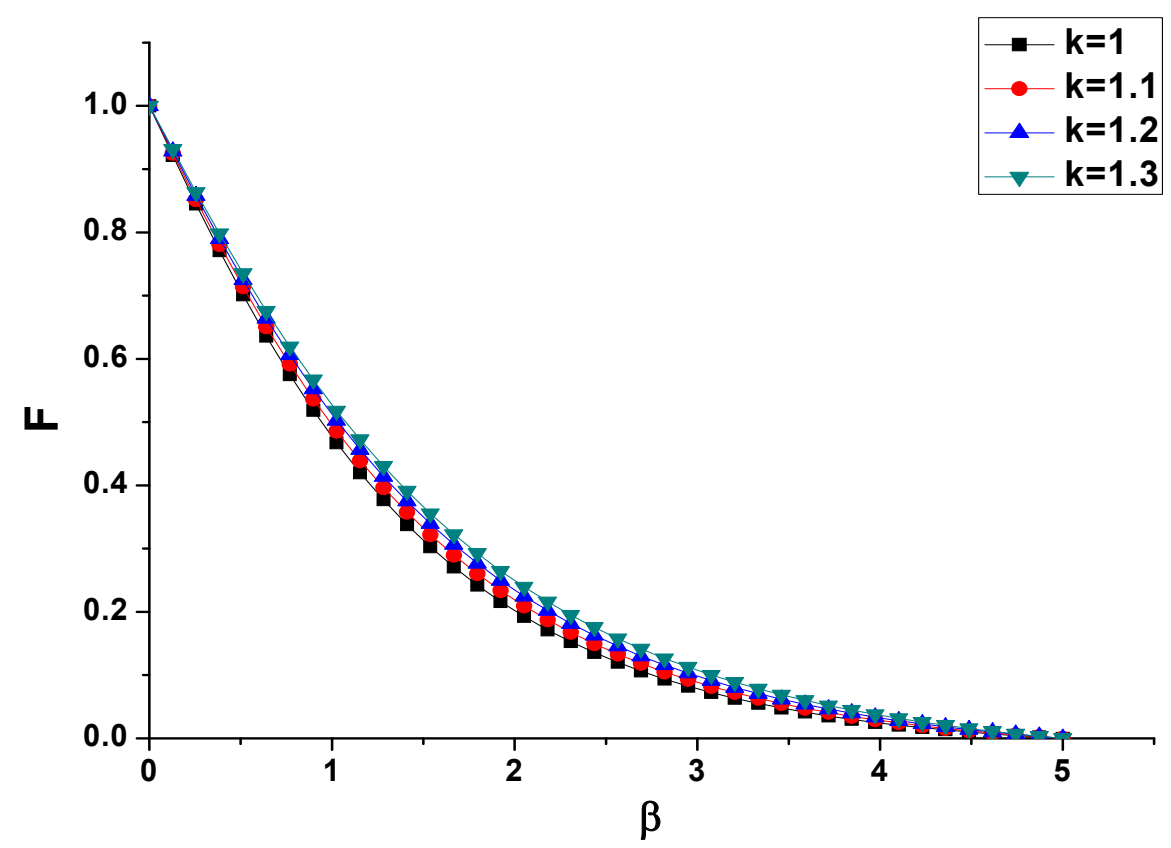

Fig.14. Steady-state tangential velocity profile for various values of $\boldsymbol{k}$. 


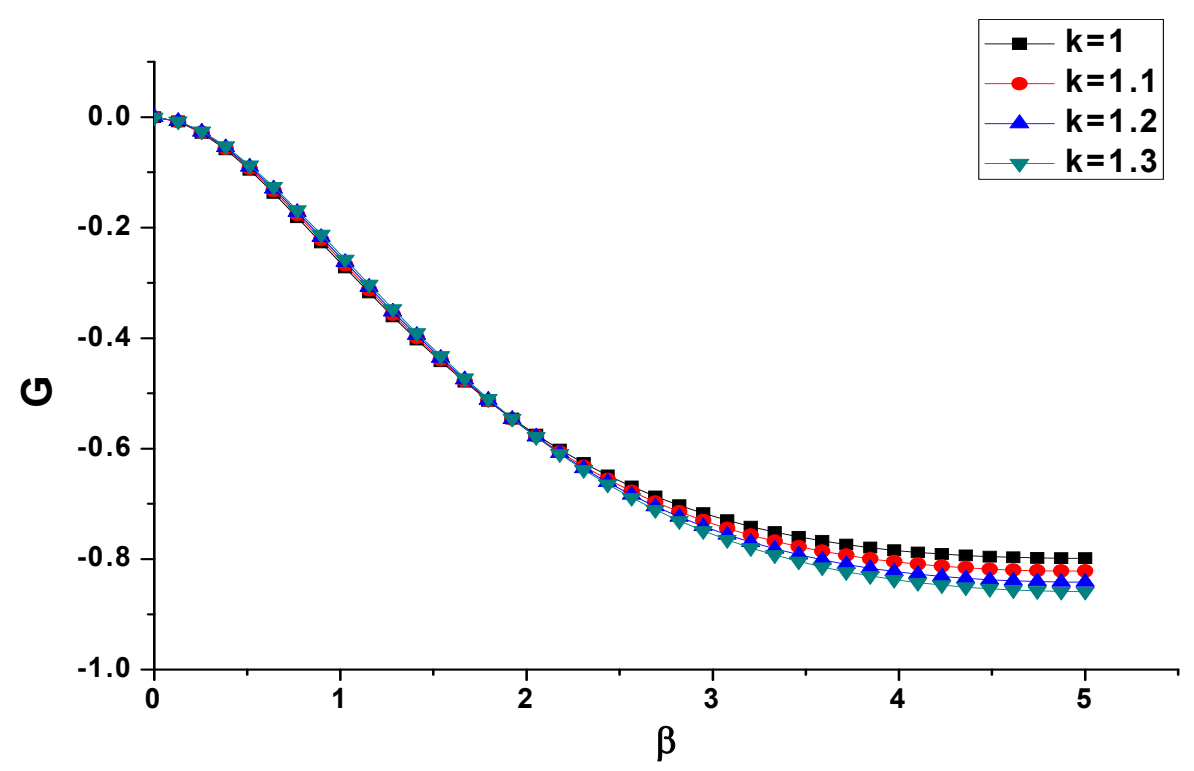

Fig.15. Steady-state axial velocity profile for various values of $\boldsymbol{k}$.

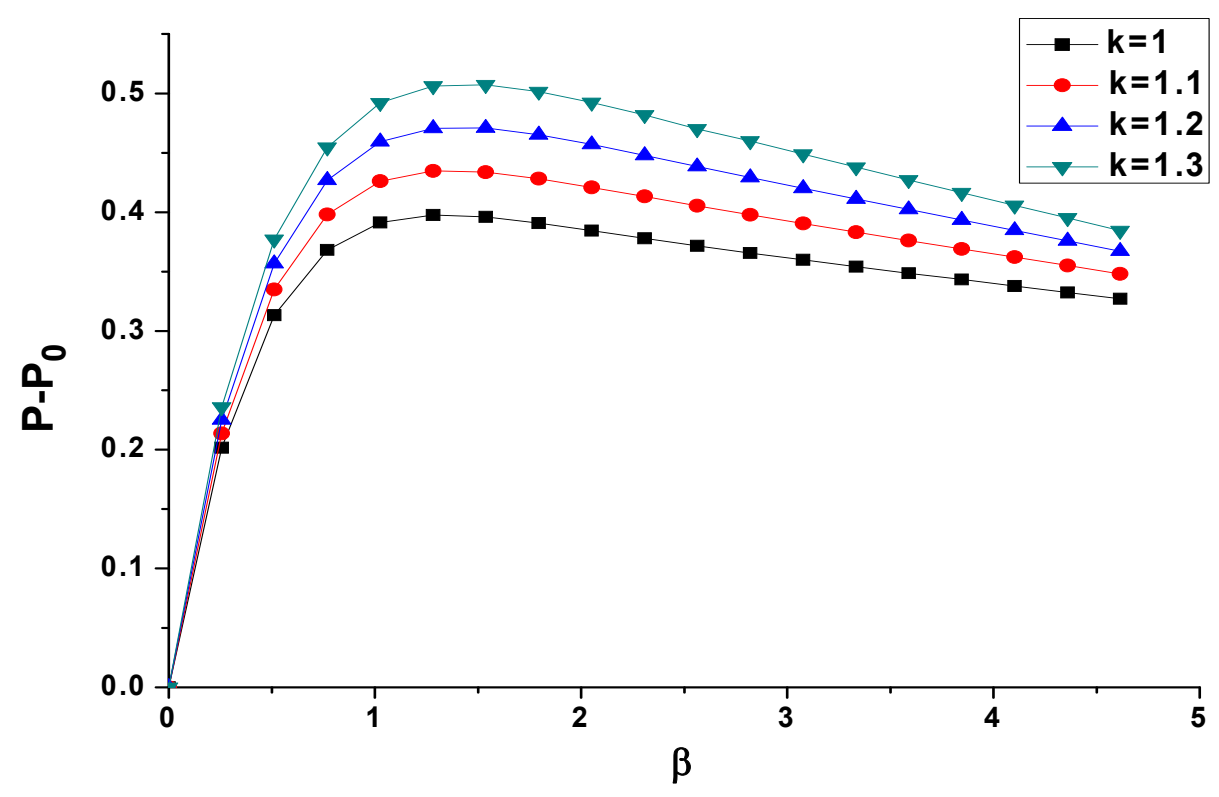

Fig.16. Pressure profile for various values of $\boldsymbol{k}$.

\section{Conclusions}

The magnetic field dependent viscosity parameter plays an important role in flow characteristics. It creates an additional resistance on the velocity distributions. Increasing magnetic field dependent viscosity increases the skin friction coefficient in ferrofluid flow. 


\section{Nomenclature}

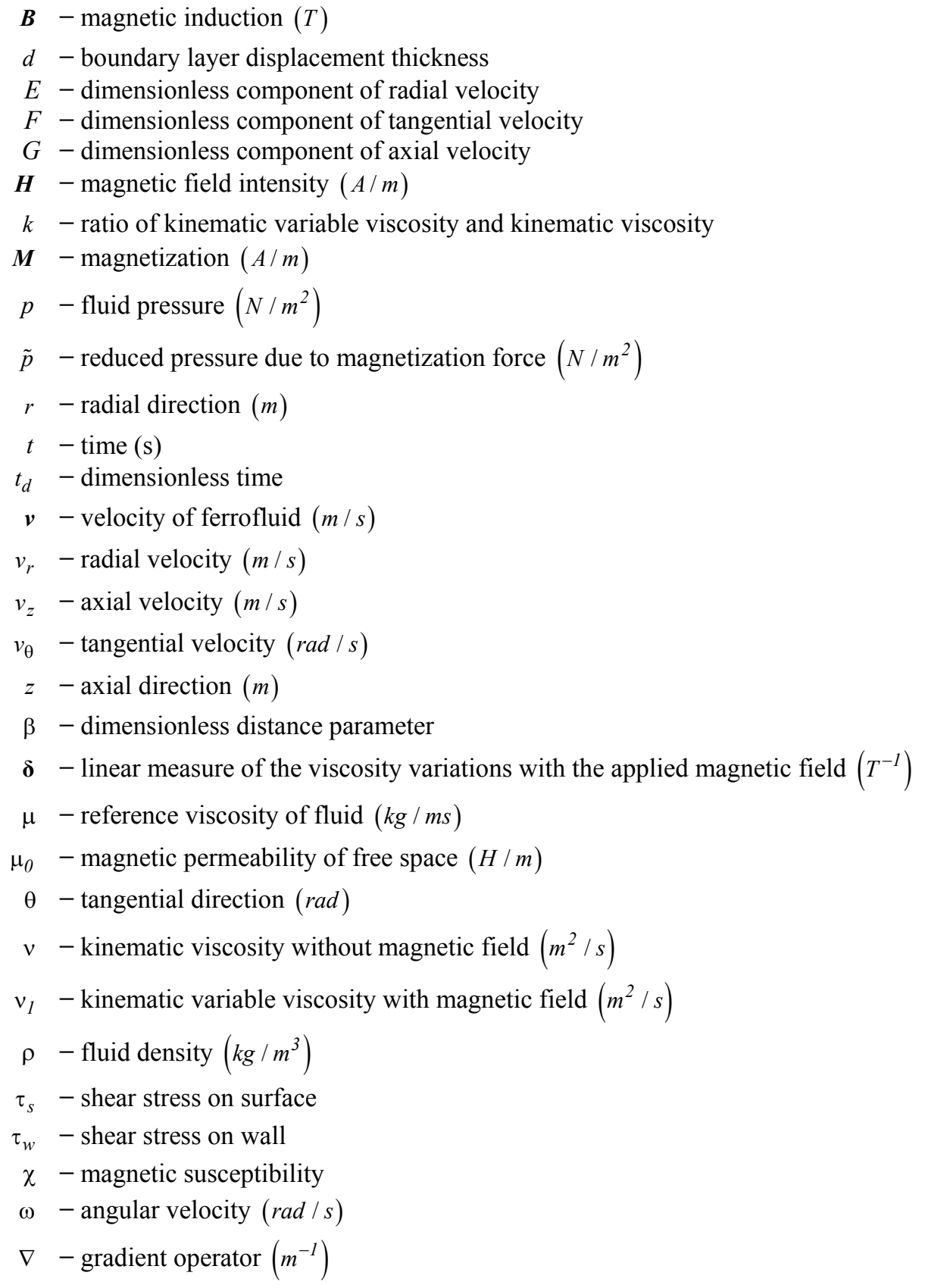

\section{References}

[1] Feynman R.P., Leighton R.B. and Sands M. (1963): Lecturers on Physics. - Addison-Wesley Reading, MA 1.

[2] Shliomis M.I. (2004): Ferrofluids as thermal ratchets. - Physical Review Letters, vol.92, No.18, 188901.

[3] Odenbach S. (2002): Magneto Viscous Effects in Ferrofluids. - Berlin: Springer-Verlag.

[4] Neuringer J.L. and Rosensweig R.E. (1964): Magnetic fluids. - Physics of Fluids, vol.7, 1927. 
[5] Verma P.D.S. and Singh M. (1981): Magnetic fluid flow through porous annulus. - Int. J. Non-Linear Mechanics, vol.16, No.3/4, pp.371-378.

[6] Verma P.D.S. and Vedan M.J. (1979): Steady rotation of a sphere in a paramagnetic fluid. - Wear, vol.52, pp.201-218.

[7] Verma P.D.S. and Vedan M.J. (1978): Helical flow of ferrofluid with heat conduction. - Jour. Math.Phy. Sci., vol.12, No.4, pp.377-389.

[8] Rosensweig R.E. (1985): Ferrohydrodynamics. - Cambridge University Press.

[9] Schlichting H. (1960): Boundary Layer Theory. - New York: McGraw-Hill Book Company.

[10] Karman V. (1921): Uber laminare and turbulente Reibung. - Z. Angew. Math. Mech. I, pp.232-252.

[11] Cochran W.G. (1934): The flow due to a rotating disc. - Proc. Camb. Phil. Sot., vol.30, pp.365-375.

[12] Benton E.R. (1966): On the flow due to a rotating disk. - J. Fluid Mech., vol.24, No.4, pp.781-800.

[13] Attia H.A. (1998): Unsteady MHD flow near a rotating porous disk with uniform suction or injection. - Journal of Fluid Dynamics Research, vol.23, pp.283-290.

[14] Mithal K.G. (1961): On the effects of uniform high suction on the steady flow of a non-Newtonian liquid due to a rotating disk. - Quart J. Mech. and Appl. Math.XIV, pp.401-410.

[15] Attia H.A. and Aboul-Hassan A.L. (2004): On hydromagnetic flow due to a rotating disk. - Applied Mathematical Modelling, vol.28, pp.1007-1014.

[16] Venkatasubramanian S. and Kaloni P.N. (1994): Effect of rotation on the thermo-convective instability of a horizontal layer of ferrofluids. - International Journal of Engineering Sciences, vol.32, No.2, pp.237-256.

[17] Belyaev A.V. and Simorodin B.L. (2009): Connvection of a ferrofluid in an alternating magnetic field. - Jour. of Applied Mechanics and Technical Physics, vol.50, No.4, pp.558-565.

[18] Sekar R., Vaidyanathan G. and Ramanathan A. (1993): The ferroconvection in fluid saturating a rotating densely packed porous medium. - International Journal of Engineering Sciences, vol.31, No.2, pp.241-250.

[19] Attia H. (2009): Steady flow over a rotating disk in porous medium with heat transfer. - Nonlinear Analysis: Modelling and Control, vol.14, No.1, pp.21-26.

[20] Frusteri F. and Osalusi E. (2007): On MHD and slip flow over a rotating porous disk with variable properties. Int. Comm. in Heat and Mass Transfer, vol.34, pp.492-501.

[21] Nanjundappy C.E., Shivakumara I.S. and Arunkumar R. (2010): Benard-Marangoni ferroconvection with magnetic field dependent viscosity. - Journal of Magnetism and Magnetic Materials, vol.322, pp.2256-2263.

[22] Ram P., Bhandari A. and Sharma K. (2010): Effect of magnetic field-dependent viscosity on revolving ferrofluid. - Journal of Magnetism Magnetic Materials, vol.322, No.21, pp.3476-3480.

[23] Ram P. and Bhandari A. (2013): Negative viscosity effects on ferrofluid flow due to a rotating disk. - Int. Journal of Applied Electromagnetics and Mechanics, vol.41, No.3, pp.467-478.

[24] Ariel P.D. (2003): On the flow of an elastic-viscous fluid near a rotating disk. - Journal of Computational and Applied Mathematics, vol.154, pp.1-25. 